\title{
Simulating accelerated atoms coupled to a quantum field
}

\author{
Marco del Rey, ${ }^{1}$ Diego Porras, ${ }^{2}$ and Eduardo Martín-Martínez ${ }^{3}$ \\ ${ }^{1}$ Instituto de Física Fundamental, CSIC, Serrano 113-B, E-28006 Madrid, Spain \\ ${ }^{2}$ Departamento de Física Teórica I, Universidad Complutense, E-28040 Madrid, Spain \\ ${ }^{3}$ Institute for Quantum Computing, University of Waterloo, 200 Univ. Avenue W, Waterloo, Ontario N2L 3G1, Canada
}

(Received 1 September 2011; published 21 February 2012)

\begin{abstract}
We show an analogy between static quantum emitters coupled to a single mode of a quantum field and accelerated Unruh-DeWitt detectors. We envision a way to simulate a variety of relativistic quantum field settings beyond the reach of current computational power, such as a high number of qubits coupled to a quantum field following arbitrary noninertial trajectories. Our scheme may be implemented with trapped ions and circuit QED setups.
\end{abstract}

DOI: 10.1103/PhysRevA.85.022511

PACS number(s): 31.30.J-, 03.67.Ac, 04.62.+v

\section{INTRODUCTION}

The study of accelerated atoms interacting with a quantum field is a fundamental problem, which has attracted a great deal of attention in the area of general relativity. Even though it has been treated extensively in the literature [1], it still poses intriguing questions, as well as experimental challenges for the detection of quantum effects induced by acceleration. Moreover, the emerging field of relativistic quantum information has recently increased the attention drawn to the topic, and in particular to the study of correlations and entanglement in noninertial scenarios [2].

Acceleration effects in quantum field theory are well understood in the perturbative regime, where, for example, transition rates are obtained for the excitation probability of atoms due to general-relativity effects. A physical paradigm in this regime is the detection of the Unruh effect [3], which, roughly, implies that an atom accelerated in the vacuum of the field is excited in the same way as an inertial atom in an effective thermal field state. Going beyond the perturbative regime poses a tough theoretical problem, which gets even harder if one considers a set of emitters, in which case we face a many-body situation, which is a situation particularly relevant from the point of view of relativistic quantum information.

In this paper, we show that a system of accelerated atoms [4] coupled to a bosonic field in the discrete mode approximation $[5,6]$ shares interesting analogies to time-dependent problems in quantum optics. Our work is motivated by the recent experimental progress that has allowed physicists to develop tools to control the dynamics of single emitters coupled to fields in setups such as trapped ions [7] or circuit QED [8]. The application of those systems as analog simulators of accelerated atoms is particularly relevant to many-body nonperturbative regimes, where numerical calculations are difficult. Furthermore, the insight gained by such analogies motivates the study of physical effects that may be relevant to the experimental detection of the Unruh effect. For example, collective phenomena such as superradiance $[9,10]$ (which are known to amplify the effective coupling of a set of emitters to the field) could be used to increase the detection sensitivity of quantum effects in noninertial scenarios.

The paper is structured as follows: We start by presenting the Unruh-DeWitt detector model to characterize an atom coupled to a quantum field. Working in the interaction picture from the comoving atom reference frame, we show how to account for a uniform acceleration in the Hamiltonian. We then proceed to discuss the possible physical implementations in trapped ions and circuit QED. For both cases, a model of emitters with controlled time-dependent atom-field couplings is presented, which, after some approximations, yields an identical Hamiltonian. For the sake of simplicity, we will refer generically to single quantum emitters as "atoms" and the bosonic mode as "field," with the latter being a phonon mode in a Coulomb crystal of trapped ions, or a photon mode confined in the cavity in circuit QED. Then, as an illustrative example, we analyze the physics in the case of a single atom to predict the outcome of simple experimental realizations of our ideas. An analogy to a decoupling process is explained in terms of the well-known Landau-Zener formula. Some future research lines on the topic will naturally emerge from our discussion.

\section{ACCELERATED UNRUH-DEWITT DETECTORS}

The Unruh-DeWitt detector $[1,4,11]$ is a standard model for a two-level atom coupled to a scalar field. This kind of detector has been extensively used for multiple purposes, such as acknowledging acceleration effects in cavities, measuring quantum correlations between spatially separated regions of spacetime, detecting entanglement degradation due to the Unruh and Hawking effects, and proposing setups to directly detect such effects. In general, computing time evolution under such a Hamiltonian is a complex problem, and thus the affordable calculations reduce to very simple scenarios where perturbation theory to the first or second order is adequate.

On the other hand, special interest has been given to the cases where the detectors couple only to a discrete number of modes. Presented in [5] and extended in [6], such settings directly model the interesting case of accelerated atoms going through a cavity. Moreover, they also feature a way to directly measure the Unruh effect considering cavities which are leaky to a finite number of modes [5].

Let us start by presenting the discretized Unruh-DeWitt interaction Hamiltonian consisting of a set of two-level atoms coupled to a scalar field. We assume a set of atoms that are accelerating with proper acceleration $a$. From the atoms perspective, the Hamiltonian can be rewritten as

$$
\begin{aligned}
H_{\mathrm{I}}= & \sum_{j m} g_{j m}\left(\sigma_{j}^{+} e^{i \Omega_{j} \tau}+\sigma_{j}^{-} e^{-i \Omega_{j} \tau}\right) \\
& \left(a_{m}^{\dagger} e^{i\left[\omega_{m} t(\tau, \xi)-k_{m} x(\tau, \xi)\right]}+a_{m} e^{-i\left[\omega_{m} t(\tau, \xi)-k_{m} x(\tau, \xi)\right]}\right),
\end{aligned}
$$


where $(\tau, \xi)$ are the proper spacetime coordinates of the accelerated detectors, and $(t, x)$ are Minkowskian coordinates. The following relation holds:

$$
c t=\xi \sinh (a \tau / c), \quad x=\xi \cosh (a \tau / c) .
$$

Directly from (2), we see that for constant $\xi$, these coordinates describe hyperbolic trajectories in spacetime whose asymptote is the light cone. A uniformly accelerated observer whose proper coordinates are (2) follows the trajectory of constant Rindler position $\xi=c^{2} / a$ [12].

If all of the detectors follow such a trajectory, then we can rewrite the Hamiltonian in a form which is suitable to find quantum optical analogs,

$$
H_{\mathrm{I}}=\sum_{j m} g_{j m}\left(\sigma_{j}^{+} e^{i \Omega_{j} \tau}+\text { H.c. }\right)\left(a_{m}^{\dagger} e^{i \Phi_{m}(\tau)}+\text { H.c. }\right),
$$

where $\tau$ is the atoms' proper time, and

$$
\Phi_{m}(\tau)=-k_{m} \xi e^{-a \tau / c}=-\frac{k_{m} c^{2}}{a} e^{-a \tau / c}=-\frac{\omega_{m}}{\alpha} e^{-\alpha \tau},
$$

where we have used the relation $\omega_{m}=c k_{m}$, and defined the parameter $\alpha=a / c$. Equation (3) defines the Hamiltonian of interest that we aim to simulate. It also reproduces a scenario where an array of detectors are resisting near the event horizon of a Schwarzschild black hole, if we consider in (3) that $a \approx$ $\kappa / \sqrt{f_{0}}$, where $\kappa$ is the surface gravity of the black hole and $f_{0}$ is the gravitational redshift factor, following the arguments in [13].

\section{PHYSICAL IMPLEMENTATIONS: TRAPPED IONS}

This setup is ideally suited to prepare and measure quantum states by well-established experimental techniques [7], which have found an important application in the quantum simulation of many-body physics [14], as well as single-particle dynamics in special relativity [15]. Previous proposals also allowed the simulation of quantum fields using trapped ions in generalrelativistic settings but in different contexts [16], however, paying the price of requiring control on the frequency of the qubit and the coupling strength. In our proposal, only phase control is needed. Our setting is therefore readily exportable to other experimental setups, as shown below.

In our scheme, we consider a chain of $N$ ions of mass $M$. For simplicity, we focus on the single-mode version of (3), and consider that the bosonic mode is a phonon mode of the chain, namely, the center-of-mass mode, which accounts for a homogeneous displacement of all the ions. State-of-the-art techniques may be used to implement phonon sidebands, and to control the atom-field coupling.

The vibrations of a Coulomb chain consist of a set of normal modes described by $H_{0}=\sum_{n} \omega_{n} a_{n}^{\dagger} a_{n}$, with $\omega_{n}$ as the normal mode frequencies, and $a_{n}, a_{n}^{\dagger}$ as the phonon operators. Levels $|0\rangle$ and $|1\rangle$ are two electronic states of the ions. Two sets of lasers couple those levels by means of Raman transitions, with amplitudes $\Omega_{\mathrm{L}, j}$, and two frequencies $\omega_{\mathrm{L}_{1,2}}$, (see [7] for details),

$$
H_{\mathrm{L}}(\tau)=\sum_{j, \nu=1,2} \frac{\Omega_{\mathrm{L}, j}}{2} \sigma_{j}^{+} e^{i k_{\mathrm{L}} x_{j}} e^{-i \omega_{\mathrm{L} \nu} \tau-i \phi_{\nu}(\tau)}+\text { H.c. }
$$

This equation represents a standard atom-light interaction term, with the only peculiarity being that we consider timedependent phases $\phi_{1}(\tau), \phi_{2}(\tau)$. Here, $x_{j}$ are operators corresponding to the ion displacements, relative to the equilibrium positions along the chain. We express those displacements in terms of phonon operators, $x_{j}=\sum_{n} \mathcal{M}_{j, n} \bar{x}_{n}\left(a_{n}+a_{n}^{\dagger}\right)$, where $\mathcal{M}_{j, n}$ are phonon wave functions, and $\bar{x}_{n}=1 / \sqrt{2 M \omega_{n}}$.

We choose laser frequencies close to resonance with the center-of-mass mode, $n=0$, such that $\omega_{\mathrm{L}_{1}}=-\omega_{0}-\Omega$, $\omega_{\mathrm{L}_{2}}=\omega_{0}-\Omega$, with $\Omega \ll \omega_{0}$. The coupling (5) simulates the quantum dynamics of an accelerated Unruh-DeWitt detector if (i) $k \bar{x}_{n} \ll 1$ (Lamb-Dicke regime), such that we can expand the exponential in powers of $\delta x_{j}$, and restrict only to linear atom-field couplings; and (ii) $\Omega_{\mathrm{L}, j} / 2 \ll \omega_{0}$, and $\left(\Omega_{\mathrm{L}, j} / 2\right) k \bar{x}_{n} \ll \omega_{0},\left|\omega_{0}-\omega_{n}\right| \neq 0$, so that we can neglect, in a rotating wave approximation (r.w.a.), all couplings to vibrational modes $n \neq 0$. This yields

$$
H_{\mathrm{L}}(\tau)=\sum_{j} g_{j} \sigma_{j}^{+} e^{i \Omega \tau}\left(a_{0} e^{-i \phi_{1}(\tau)}+a_{0}^{\dagger} e^{-i \phi_{2}(\tau)}\right)+\text { H.c. },
$$

where we have used that the center-of-mass vibrational modes fulfill $\mathcal{M}_{j, 0}=1 / \sqrt{N}$, such that $g_{j}=i \Omega_{\mathrm{L}_{j}} k \bar{x}_{0} /(2 \sqrt{N})$. By choosing phases such that $\phi_{1}(\tau)=-\phi_{2}(\tau)=\Phi(\tau)$, we arrive at the single-mode version of Eq. (3). Including more modes would require additional lasers on resonance with other vibrational modes. Position-dependent couplings $g_{j}$ and frequencies $\Omega_{j}$ may be achieved given a certain intensity profile of the lasers, and by focusing multiple lasers on each ion, respectively.

Typical experimental values are $\omega_{0},\left|\omega_{n}-\omega_{0}\right| \approx 1 \mathrm{MHz}$, and $k \bar{x}_{0} \approx 0.2$, such that our requirements are fulfilled with values $\Omega_{\mathrm{L}} / 2=100 \mathrm{kHz}$, and $\Omega=100 \mathrm{kHz}$, yielding $g=$ $20 / \sqrt{N} \mathrm{kHz}$. Those values are well above typical decoherence rates in trapped ions. Also, in order to observe analogs to acceleration, $\Phi(\tau)$ has to increase exponentially over a time scale comparable to the inverse energies involved in the setup. For example, values $\alpha=10^{-3} \Omega=0.1 \mathrm{kHz}$ would require one to vary the optical phase on times scales of $10 \mathrm{~ms}$. This is technically feasible by using, for example, acoustooptical modulators and standard experimental techniques from trapped ion quantum computation, which indeed require manipulation on a much shorter time scale [17]. Our ideas may also be used with spin many-boson models, as in [18].

\section{PHYSICAL IMPLEMENTATIONS: CIRCUIT QED}

Our second proposed implementation consists of a superconducting qubit coupled to a microwave cavity in the strong-coupling regime [8]. The interest in these kinds of setups has been growing in the last years, with very interesting proposals to simulate other relativistic phenomena, such as the dynamical Casimir effect in [19] or Hawking radiation [20]. In particular, a review of these vacuum amplification processes, and their possible realizations in superconducting circuit setups, has recently been done [21]. The simulation we propose in this paper is different from those in the scenario studied, including the kind of effects and the experimental idea behind it, which in our case relies on the driving of the qubit 
frequency only, and which is particularly well suited [22]. The qubits and field noninteracting Hamiltonian is

$$
H_{0}(\tau)=\omega_{0} a^{\dagger} a+\frac{\epsilon}{2} \sum_{j} \sigma_{z, j}+H_{\mathrm{d}}(\tau),
$$

where $\omega$ is the resonant frequency of the mode, and $\epsilon$ is the qubit energy. $H_{\mathrm{d}}(\tau)$ describes a driving field, for which we assume the following form:

$$
H_{\mathrm{d}}(\tau)=-\sum_{j} \sum_{\nu=1,2} \eta_{j} \omega_{\mathrm{d}_{\nu, j}} \cos \left[\omega_{\mathrm{d}_{\nu, j}} t+\phi_{\nu}(\tau)\right] \sigma_{z, j} .
$$

Note that Eq. (7) is written as a periodic driving with a phase, $\phi_{\nu}(\tau)$, which can be considered to evolve slowly in time, in a sense to be quantified below. The qubit-cavity coupling in the Schrödinger picture is given by $H_{\mathrm{I}}=g_{0}\left(\sigma^{+}+\sigma^{-}\right)\left(a+a^{\dagger}\right)$. We write the coupling in the interaction picture with respect to $H_{0}(\tau)$ as

$$
\begin{aligned}
& H_{\mathrm{I}}(\tau)=g_{0} \sum_{j}\left[\sigma_{j}^{+} e^{i \epsilon \tau} \mathcal{G}_{j}(\tau)+\text { H.c. }\right]\left(a e^{-i \omega_{0} \tau}+\text { H.c. }\right), \\
& \mathcal{G}_{j}(\tau)=e^{-2 i \sum_{v} \eta \sin \left[\omega_{d_{\nu, j}} \tau+\phi_{\nu}(\tau)\right]},
\end{aligned}
$$

where we made the approximation that $\dot{\phi}(\tau) / \phi(\tau) \ll \omega_{\mathrm{d}_{v, j}}$. Consider now that $\eta \ll 1$, and the choice of frequencies $\omega_{\mathrm{d}_{1, j}}=$ $\epsilon-\Omega_{j}-\omega_{0}, \omega_{\mathrm{d}_{2, j}}=\epsilon-\Omega_{j}+\omega_{0}$, and conditions $\epsilon, \omega_{0}, \mid \epsilon-$ $\omega_{0} \mid \gg g_{0}$. We expand $\mathcal{G}(\tau)$ to the first order in $\eta_{j}$, and keep only slowly rotating terms in a r.w.a:

$$
H_{\mathrm{I}}(\tau) \approx \eta g_{0} \sum_{j} \sigma_{j}^{+}\left(e^{i \Omega_{j} \tau-i \phi_{1}(\tau)} a+e^{i \Omega_{j} \tau-i \phi_{2}(\tau)} a^{\dagger}\right)+\text { H.c. }
$$

This expression takes the form (3) by choosing $\phi_{1}(\tau)=$ $\Phi(\tau), \phi_{2}(\tau)=-\Phi(\tau)$, and $g_{j}=g_{0} \eta_{j}$.

In circuit QED, the high-energy scales $\epsilon, \omega_{0}$ are in the $\mathrm{GHz}$ regime. Low-energy scales, such as $g_{j}, \Omega_{j}$, then may be in the $\mathrm{MHz}$ range. Finally, note that $\dot{\phi}(\tau) / \phi(\tau)=\alpha$. Thus, in order to observe the effects from effective acceleration, our scheme requires $\alpha$ on the frequency scale of $g_{j}, \Omega_{j}$, such that the driving fields in (7) have to be controlled with an inverse time in the $\mathrm{MHz}$ range. This seems technically feasible, since this rate is slower than the typical evolution times in circuit-QED systems. By using different photonic modes in a cavity, as well as local control of qubit couplings, the full multimode Hamiltonian (3) may be implemented.

\section{SINGLE DETECTOR CASE: NONADIABATIC EFFECTS INDUCED BY ACCELERATION}

We will study a simple case with a twofold purpose in mind: on the one hand, it will serve to gain some insight into the parameters required for our experimental proposal, while, on the other hand, it will unveil an analogy between nonequilibrium physics and quantum effects induced by acceleration. Let us consider a single atom $A$, with natural frequency $\Omega$, and uniform acceleration frequency $\alpha=a / c=f_{a} \Omega$, coupled to a single-mode field with proper frequency, $\omega>\Omega$.

Let us have $A$ excited at $\tau=0$ with no excitations in the field. Representing the free eigenstates of atom $(A)$ and field $(F)$ with the notation $|\psi\rangle=|A F\rangle$, the initial state would then be $|i\rangle=|e 0\rangle$. We let the system evolve naturally and

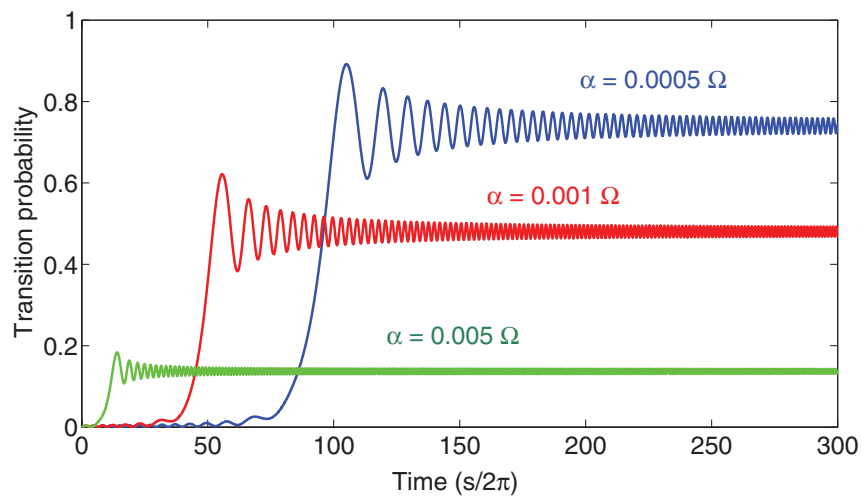

FIG. 1. (Color online) Time evolution of $\mathcal{P}_{A_{g}}$ for different accelerations, with the parameter values $g=0.01 \Omega$ and $\omega_{0}=1.33 \Omega$. The crossing time and the transition probability fit the Landau-Zener prediction described in (12).

concentrate on the probability for $A$ to decay to its ground state:

$$
\mathcal{P}_{A_{g}}(\tau)=\sum_{F}|\langle g F|U(\tau)| e 0\rangle|^{2} .
$$

It is convenient to analyze the phase in the Hamiltonian (3). The latter is equivalent to a Hamiltonian in the interaction picture with respect to a time-dependent effective frequency,

$$
\omega_{\text {eff }}(\tau)=\partial_{\tau} \Phi(\tau)=\omega_{0} e^{-\alpha \tau},
$$

which decreases asymptotically. We can, therefore, picture this situation as a system starting in a certain low-energy state, in this case matching $|e 0\rangle$, coupled to a high-energy state, which initially happens to be $|g 1\rangle$. The difference between the two effective energies varies with time as

$$
\Delta E_{\mathrm{eff}}(\tau)=\omega_{0} e^{-\alpha \tau}-\Omega,
$$

and there will be a level crossing, corresponding to a stationary $\Phi(\tau)$, taking place at $\tau_{c}=\ln \left(\omega_{0} / \Omega\right) / \alpha$.

After a long time (as compared to $1 / \alpha$ ), we can obviously approximate the low-energy level of the system as $|g 1\rangle$, and the high-energy level as $|e 0\rangle$. We can, therefore, establish an analogy between this kind of phenomenon and a typical Landau-Zener transition, making a link between nonequilibrium physics and quantum field theory in curved spacetimes. If the energy difference happens to vary very slowly (which will be the case if $\alpha \ll g$ ), then we would expect no transition between eigenstates to take place, so the system will stay in the ground state, which means actually decaying into $|g 1\rangle$. If, however, the evolution happens to be diabatic, the probability of transiting into a high excited state (i.e., staying in $|e 0\rangle$ ) can be approximated by the Landau-Zener formula,

$$
\mathcal{P}_{A_{e(+\infty)}} \simeq e^{-2 \pi \Gamma} \Rightarrow \mathcal{P}_{A_{g(+\infty)}} \simeq 1-e^{-2 \pi \Gamma}
$$

where $\Gamma=g^{2} /\left(\left.\partial_{\tau} \Delta E\right|_{\tau=\tau_{c}}\right) . \mathcal{P}_{A_{g}}$ is plotted in Fig. 1 .

We note several differences with the original Landau-Zener (L-Z) model [23]. Namely, (11) is not linear but exponential in time; also, in our case, only for small couplings, we have a twolevel problem (a r.w.a. approximation cannot be performed). Nevertheless, in the regimes considered, L-Z is a very good approximation, as shown in Fig. 2 


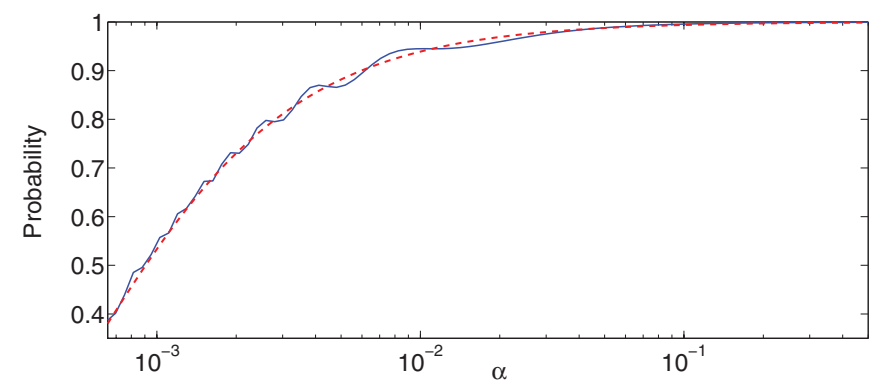

FIG. 2. (Color online) Comparison between the Landau-Zener theoretical prediction (red dashed line) and the probability for an accelerated detector of remaining in the excited state as a function of the acceleration $a=f_{\alpha} c \Omega$ (blue solid line), with parameter values $g=0.01 \Omega$ and $\omega_{0}=1.33 \Omega$.

The atom stays excited until the phase variation rate slows down at times close to $\tau_{c}$. Around that moment, the coupling terms get resonant and the decay probability grows notably. From then on, the atom and field get progressively decoupled again with the probability stabilizing itself.

\section{MANY EMITTERS CASE}

In this paper, we have given details regarding the single emitter case. However, the setting presented is readily extendible to more general and interesting cases: the Hamiltonian (1) can be simulated in a system of trapped ions taking into account that the number of qubits in our simulator should be equal to or greater than the number of simulated emitters.

With these experimental constructions, we would be able to explore many-body collective phenomena (such as superradiance), which can amplify the effective coupling of a set of emitters to the field, effectively increasing the detection sensitivity of quantum effects in noninertial scenarios: We can use these simulators to go beyond the computational limit of classical computers in order to explore collective phenomena that may amplify the relativistic effects imprinted in arrays of many detectors undergoing relativistic motion, and use what we learn in the simulator to export it to the direct detection of the Unruh and Hawking effects in both analog and real general-relativistic settings.
To do that, we depend on the current technology of the quantum control of systems of many qubits and our ability to couple them to the same quantum field. In trapped ions, the required control of systems of more than 10 ions can be achieved with current technology [24]. Considering systems of more than 10 qubits is already far beyond the reach of standard classical computer clusters, which have difficulties simulating systems of more than five emitters; this exemplifies the convenience of developing this kind of quantum simulator.

\section{CONCLUSIONS}

We have presented a scheme for simulating a set of accelerated atoms coupled to a single-mode field. First, we have identified the Hamiltonian which yields the evolution as seen by a comoving observer with the atoms. A method has been presented to obtain the same Hamiltonian for circuit QED and trapped ions by inducing time-dependent sidebands in atom-field couplings. Our idea may be extended to many-particle experiments, which could simulate results that are not affordable for classical computers, such as arbitrary noninertial trajectories of detectors and many detectors coupled to quantum fields. Finally, by interpreting our results from a Landau-Zener perspective, we have made a connection between nonequilibrium physics and quantum effects due to acceleration. This highlights an idea that will undoubtedly be exploited in the future, and which has already been explored in recent works [25] in a context quite different to this one: general-relativistic quantum effects can be studied with tools coming from nonequilibrium physics.

\section{ACKNOWLEDGMENTS}

The authors thank M. Montero for his helpful comments, and J. J. García-Ripoll for discussions. E.M-M. and M.dR. were supported by a CSIC JAE-PREDOC grant. M.dR. was also supported by Residencia de Estudiantes. D.P. was supported by RyC Contract No. Y200200074 and EU (PICC), Contracts No. FIS2009-10061 and No. CAM-UCM/910758. The authors were partially supported by QUITEMAD Project No. S2009-ESP-1594.
[1] L. C. B. Crispino, A. Higuchi, and G. E. A. Matsas, Rev. Mod. Phys. 80, 787 (2008); A. Higuchi, G. E. A. Matsas, and D. Sudarsky, Phys. Rev. D 46, 3450 (1992).

[2] B. Reznik, A. Retzker, and J. Silman, Phys. Rev. A 71, 042104 (2005); I. Fuentes-Schuller and R. B. Mann, Phys. Rev. Lett. 95, 120404 (2005); S.-Y. Lin and B. Hu, Found. Phys. 37, 480 (2007); S.-Y. Lin, C.-H. Chou, and B. L. Hu, Phys. Rev. D 78, 125025 (2008); S.-Y. Lin and B. L. Hu, Classical Quantum Gravity 25, 154004 (2008); A. G. S. Landulfo, G. E. A. Matsas, and A. C. Torres, Phys. Rev. A 81, 044103 (2010); I. Fuentes, R. B. Mann, E. Martín-Martínez, and S. Moradi, Phys. Rev. D 82, 045030 (2010).

[3] W. G. Unruh, Phys. Rev. D 14, 870 (1976).

[4] DeWitt, General Relativity: An Einstein Centenary Survey (Cambridge University Press (Cambridge, UK, 1980).
[5] E. Martín-Martínez, I. Fuentes, and R. B. Mann, Phys. Rev. Lett. 107, 131301 (2011).

[6] A. Dragan and I. Fuentes, e-print arXiv:1105.1192v2.

[7] D. Leibfried, R. Blatt, C. Monroe, and D. Wineland, Rev. Mod. Phys. 75, 281 (2003).

[8] A. Wallraff, D. I. Schuster, A. Blais, L. Frunzio, R.-S. Huang, J. Majer, S. Kumar, S. M. Girvin, and R. J. Schoelkopf, Nature (London) 431, 162 (2004).

[9] M. Gross and S. Haroche, Phys. Rep. 93, 301 (1982).

[10] P. Nataf and C. Ciuti, Nat. Commun. 1, 72 (2010).

[11] J. Louko and K. Schleich, Classical Quantum Gravity 16, 2005 (1999); 23, 6321 (2006); J. Louko and A. Satz, ibid. 25, 055012 (2008).

[12] C. W. Misner, K. S. Thorne, and J. A. Wheeler, Gravitation (Freeman, San Francisco, 1973). 
[13] E. Martín-Martínez, L. J. Garay, and J. León, Phys. Rev. D 82, 064006 (2010).

[14] C. Schneider, D. Porras, and T. Schaetz, e-print arXiv:1106.2597.

[15] R. Gerritsma, G. Kirchmair, F. Zähringer, E. Solano, R. Blatt, and C. F. Roos, Nature 463, 68 (2010).

[16] P. M. Alsing, J. P. Dowling, and G. J. Milburn, Phys. Rev. Lett. 94, 220401 (2005); N. C. Menicucci, S. J. Olson, and G. J. Milburn, New J. Phys. 12, 095019 (2010).

[17] D. Leibfried, R. Blatt, C. Monroe, and D. Wineland, Rev. Mod. Phys. 75, 281 (2003).

[18] D. Porras, F. Marquardt, J. von Delft, and J. I. Cirac, Phys. Rev. A 78, 010101 (2008).
[19] J. R. Johansson, G. Johansson, C. M. Wilson, and F. Nori, Phys. Rev. Lett. 103, 147003 (2009).

[20] P. D. Nation, M. P. Blencowe, A. J. Rimberg, and E. Buks, Phys. Rev. Lett. 103, 087004 (2009).

[21] P. D. Nation, J. R. Johansson, M. P. Blencowe, and F. Nori, Rev. Mod. Phys. 84, 1 (2012).

[22] D. Porras and J. José García-Ripoll, e-print arXiv:1107.2607.

[23] C. Zener, R. Soc. London Proc. Ser. A 137, 696 (1932).

[24] T. Monz, P. Schindler, J. T. Barreiro, M. Chwalla, D. Nigg, W. A. Coish, M. Harlander, W. Hänsel, M. Hennrich, and R. Blatt, Phys. Rev. Lett. 106, 130506 (2011).

[25] P. Nation, M. P. Blencowe, and F. Nori, e-print arXiv:1009.3974. 